
STRUCTURES

Samer Tawfik, Georgia Institute of Technology

Serkan Ozbay, Georgia Institute of Technology

Erian Armanios, Georgia Institute of Technology 


\section{An Effective Approach for Teaching Aerospace Structures}

\section{I- Abstract}

This paper discusses a method aiming at providing tailored education that would increase students understanding of the material and enhance their success. The method is being applied to COE 3001 (Mechanics of Deformable Bodies), the second structures course in Aerospace Engineering undergraduate program at the Georgia Institute of Technology. COE 2001 (Statics) is the first class in structures and introduces students to the elements of statics in two and three dimensions, free-body diagrams, distributed loads, centroids and friction. In COE 3001 the students are further exposed to stress and strain analysis applied to beams, vessels, pipes, and combined loading, stress and strain transformations, beam deflection as well as column buckling.

The method consists of weekly problem sessions, a test evaluation concept and a tailored workplan. The weekly problem sessions are conducted by teaching assistants. The students are given a chance to discuss with the TA a set of suggested textbook problems assigned by the instructor as well as any other recommended problems pertaining to the topics covered in the lectures. These include practical problems that are recommended by the instructor as bonus problems. The students are encouraged to submit their work on the suggested problems a few days after the problem sessions for evaluation by the TA. During this period the TA maintain an open-door policy where students can have one-on-one interaction.

The test evaluation concept provides a break down of the main elements relevant to the basic concepts in mechanics of deformable bodies such as free body diagrams, shear force bending moment diagrams or stress and strain transformations. These elements are used for an individual student evaluation. Based upon these evaluations a tailored work-plan consists of sets of problems are suggested to each student to address a weakness or deficiency in given fundamental concepts.

\section{II- Introduction}

Spence $^{1}$ focused on showing the importance of preparing students for today's work world. One of the major skills students should have before getting a job is the ability for continuous learning. Such a skill can not be obtained through traditional methods of teaching; hence "learning" should be the ultimate goal. Its outcome will be a student who is well prepared to learn and think critically.

Marshall and Marshall ${ }^{2}$ introduced five learning techniques, one of which is "Problem Based Learning". This technique also known as problem solving learning would help students to develop skills, such as: Critical thinking, and ability to analyze complex problems, Communication skills and ability to cooperate, Research, explore and evaluate resources and Continuous learning personality. 
A weekly problem session is designed to serve the purpose of "Problem Based Learning". Students discuss their ideas with each other and/or with the TAs. As a result a homework assignment is not a secluded responsibility anymore but a collective experience. The details of implementing this technique are presented in section 3.

Frequently instructors are faced with a student concerning "I didn't do well in the last midterm and I'm worried about my grades". In most cases, instructors can't help such a student even if they wanted; simply a student has to help himself/herself. Hence the following concept is implemented into teaching Aerospace Structures, "Test Evaluation Concept". There are many advantages to this concept for the instructor and the students:

- It allows the instructor to monitor students' progress throughout the class

- It provides a feedback through specific corrective actions so the focus switches to the remedy phase rather than a prolonged shortcoming phase

- It allows a "one on one" education environment between the instructor and each student. This concept is a departure from traditional classroom teaching. Goankar ${ }^{3}$ pointed out the importance of output feedback in order to enhance the teaching process. On top of returning a graded test to the students, they receive an evaluation of their effort. Their weaknesses are isolated and methods to remedy any deficiency are suggested.

\section{III- Problem-Based Learning, weekly problem session}

Problem-based learning is applied in medical schools and forms a main learning strategy. Evenson and $\mathrm{Hmelo}^{4}$ underscore the vitality of such a strategy for medical students by instituting learning around a series of biomedical problems presented in small groups with the faculty functioning as "tutors or guides to learning".

Weekly problem sessions are designed to serve the purpose of problem based learning. These sessions are conducted by TAs functioning as guides to learning. During these sessions, students discuss and express their ideas concerning a set of suggested problems. Two main categories of problems are discussed in these weekly sessions assigned homework problems and extra credit or bonus problems. The instructor of the class designs problems in each category while the TAs are responsible for guiding the students through solution procedures, conclusions and practical application.

A- Assigned homework problems

These are selected from the textbook with varying degrees of complexity. They include some simple problems to stress straightforward application of concepts or theories explained in lecture. More complex problems will require to be broken down into a set of simpler elements or tasks. Being exposed to such problems students build the ability to critical thinking.

Students are asked to present their suggestions and questions to solve each problem. The logic behind each suggestion and/or question is discussed by the TAs in a way that motivates the students to follow the same logic in solving a problem. Conclusions and interpretations of the results obtained for each problem are presented such that the objective of the problem becomes clearer. 
B- Extra credit and/or bonus problems

These problems cover the practical aspect of applying the theory. Real life problems are discussed showing the application of the theory in the process of the design of an aircraft. Presentations by guest speakers from industry illustrate this category. An invited presentation ${ }^{5}$ exposed students to a practical use of shear force and bending moment diagrams to demonstrate how the addition of pylon loads at span-wise stations helps relieve shear loading and bending moment in the wing. Students were asked to write a report following these presentations and encouraged to continue their inquiries through email questions to the presenter. One student conclusion to this presentation ${ }^{5}$ seminar is representative of the gained benefits "Overall, this seminar was very interesting and definitely gave me a perspective on how structural analysis is used in the aerospace field. I was able to see how structural analysis fits in the big picture with other aspects of aerospace engineering (such as vehicle dynamics and aerodynamics), and I was also able to learn about work currently being performed and tools currently being used in the field. Attending Mr. Sharp's presentation was definitely a worthwhile experience for me"

In summary weekly problem sessions help students to

a- Build the ability to analyze complex problems through mastering critical thinking

b- Enhance communication skills and ability to cooperate

c- Introduce excitement of research

d- Adopt a continuous learning path

\section{IV- Test Evaluation Concept}

Educational research shows that teaching in the form of lectures is no more than transmitting information. Shannon and Weaver's model of communication, shown in Figure 1, is often used to represent lecture halls teaching as an open loop system.

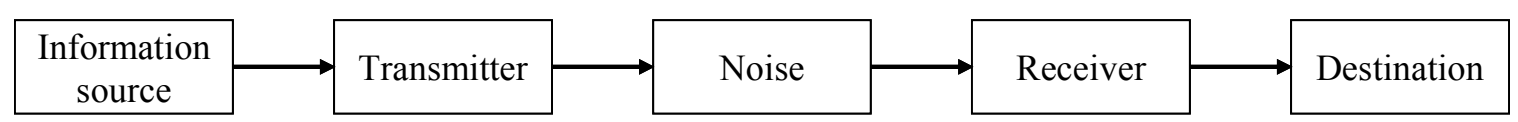

Figure 1- Shannon-Weaver's Communication Model

Goankar [3] suggested that Shannon and Weaver's model with added feedback can enhance the teaching-learning process. In terms of engineering system a portion of the output is fed back to the input as shown in Figure 2.

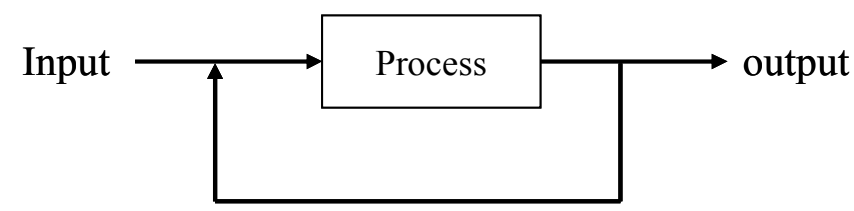

Figure 2- Engineering System Model with feedback 
A main difference between the engineering system model and the suggested modification of the Shannon-Weaver's Communication system lies within the nature of the feedback. In the engineering system model, the function of the feedback is to maintain the output of the system constant, i.e. establish equilibrium. While in the modified version of Shannon-Weaver's model the feedback is of a corrective nature and works continuously to increase the output.

Traditional methods used by instructors to obtain feedback include asking questions during lectures, assigning homework sets and utilizing quizzes and tests. Feedback in these cases works as a good indicator of students' progress but its goal in terms of a corrective action is not being completely utilized. Engineering systems in most cases make use of controllers in order to obtain higher output accuracy as seen in Figure 3. This in turn requires analyzing the feedback signal to make it more useful in providing a tuned output at higher precision.

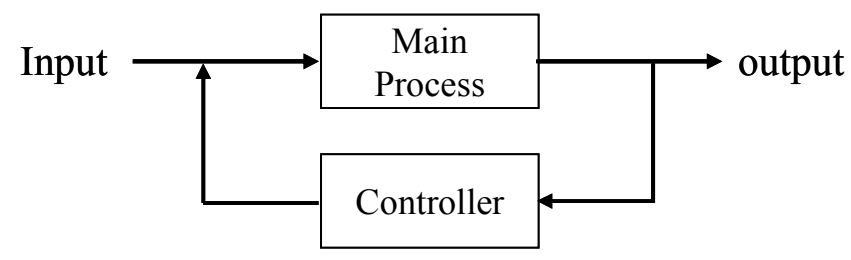

Figure 3- Engineering System Model with controlled feedback

The Shannon-Weaver's communication model is now modified to include a controlled feedback signal. This controlled feedback signal is referred to in the present approach to teaching Aerospace Structures as "Test Evaluation Concept". It should be noted that the "Test Evaluation Concept" can be adapted to teaching any materials. This evaluation can be performed while grading each student paper by

a- Breaking down a problem into a number of fundamental concepts being tested.

b- Evaluating students' proficiency in determining each specified concepts tested in diverse configurations

c- Providing a corrective action (through suggesting a set of principles review problems and examples from the textbook) to rectify any deficiency if needed

The "Test Evaluation Concept" will give feedback related to gained knowledge in lectures and weekly problem sessions. It also reflects each student understanding of basic and their progress throughout the class. 
An illustration of the evaluation concept is provided by one problem assigned in a midterm test and shown in Figure 4.

A beam with the $\mathrm{T}$ cross section is under a concentrated force and a uniformly distributed load as shown in figure, Determine

- Maximum axial stress in the cross-section and its location

- Maximum shear stress in the cross-section and its location

If a tensile force of $2 \mathrm{KN}$ is applied at end ' $\mathrm{B}$ ' of the beam, find the location of the "Neutral Axis" at section ' $\mathrm{C}$ '
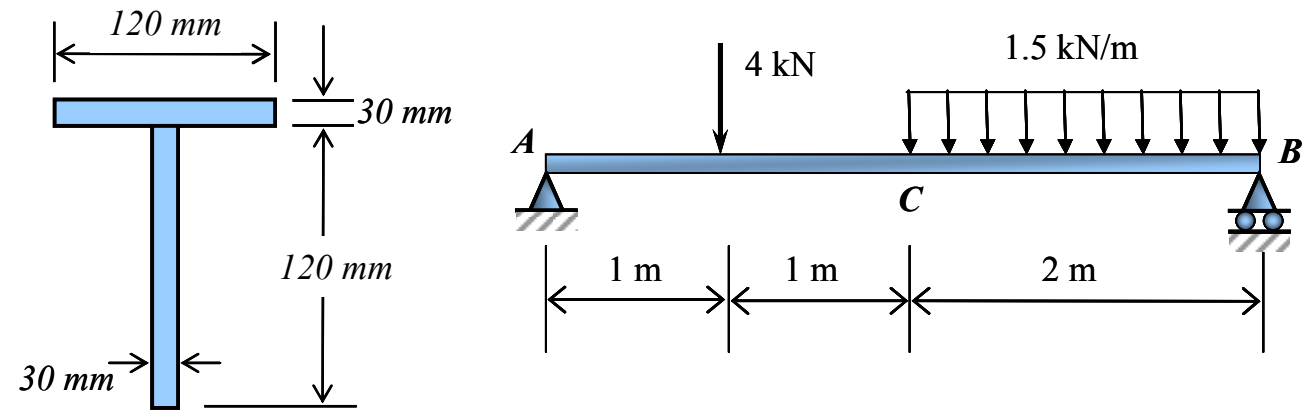

Figure 4- Example problem

The evaluation sheet for this problem is shown in Figure 5. A copy of this sheet is attached to the student's solution while the original is kept by the instructor. The sheet consists of a table with a number of rows corresponding to the elements tested, the columns encompass credit allocated and received for each element, and an explanation (showing a reason of error if any). The instructor may also include an entry of required corrective actions to rectify any deficiency at the bottom of the table. Corrective action maybe in the form of assigned problems from the textbook specially selected to obtain rectification.

A copy of this evaluation sheet is given to students at the same time they receive their graded tests. Thus help some to switch their focus from disappointment over their shortcomings to remedy through working the corrective actions. As a follow-up students are encouraged to address the suggested corrective actions for a bonus. 


\begin{tabular}{|c|c|c|c|c|}
\hline & \multirow{2}{*}{ Item } & \multicolumn{2}{|c|}{ Credit } & \multirow{2}{*}{ Reason } \\
\hline & & Allocated & Received & \\
\hline 1 & Reactions & 5 & .5 & 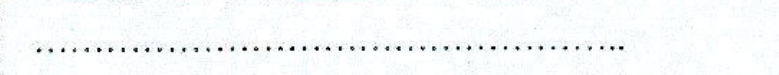 \\
\hline 2 & $\mathrm{~V}_{\mathrm{MAX}}$ & 5 & .3. & 7. Eq. s...parthy risht ........ \\
\hline 3 & $\mathbf{M}_{\mathrm{MAX}}$ & 5 & .3 & ...... \\
\hline 4 & $\begin{array}{c}\text { Neutral Axis } \\
\mathbf{y}_{\text {N.A. }}\end{array}$ & 5 & ... & Bidna't ...prourde \\
\hline 5 & I (parallel axis) & 10 & $.5 \ldots$ & No .......fl... Gxis thamp used \\
\hline 6 & $Q$ & 5 & $\ldots 2$ & wroge Equs ........................... \\
\hline 7 & $\sigma_{\mathrm{MAX}}$, location & 10 & .2 & Fust Eqn .....mo sohn.... \\
\hline 8 & $\tau_{\mathrm{MAX}}$, location & 10 & .3. & $\begin{array}{l}\text { Tunt Equ and unong Numbers. } \\
\qquad \& \text { solw. }\end{array}$ \\
\hline 9 & $\begin{array}{l}\text { New Neutral } \\
\text { Axis } \mathbf{y}_{\text {NEw. }}\end{array}$ & 5 & (1). & 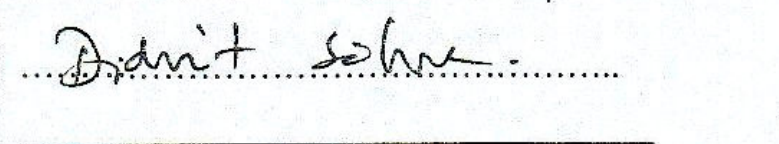 \\
\hline & & 60 & 23 & \\
\hline
\end{tabular}

Figure 5- Filled evaluation sheet

The filled evaluation sheet shown in Figure 5 identified a number of deficiencies for this student, such as not being able to find correct shear force and bending moment distribution. The student failed to show understanding in regards to the Neutral Axis. A number of problems were assigned to this particular student as corrective actions. The student is encouraged to work on these problems and discuss them with the TAs during office hours. 
Sample test statistical data are provided in Table 1. These statistical measures are incorporated into the instructor's lectures to address gaps in students understanding. Also included in the table a comparison of False/True type question as a measure of students mastery of the same fundamental concepts. Interestingly False/True questions underestimated student mastery $(71.25 \%$ versus $76 \%)$ of shear force diagrams and overestimated bending moment diagram $(61.2 \%$ versus $36 \%)$.

Table 1. Sample test statistical data

\begin{tabular}{lc} 
Correct Responses based on write-in solutions to 3 problems \\
\hline Reactions & $40 / 66$ or $61.0 \%$ \\
Shear Force Diagram & $50 / 66$ or $76.0 \%$ \\
Bending Moment Diagram & $24 / 66$ or $36.0 \%$ \\
Centroid & $41 / 66$ or $62.0 \%$
\end{tabular}

Comparison based on TRUE/FALSE questions

Shear Force Diagram

Problem 1:

$50 / 60$ or $83.3 \%$

Problem 2:

$45 / 60$ or $75.0 \%$

Problem 3:

$34 / 60$ or $56.6 \%$

Problem 4:

$42 / 60$ or $70.0 \%$

Average $\quad \mathbf{7 1 . 2 5 \%}$

Bending Moment Diagram

Problem 1:

$39 / 60$ or $65.0 \%$

Problem 2:

$33 / 60$ or $55.0 \%$

Problem 3:

Average

$62.2 \%$

$40 / 60$ or $66.6 \%$

Further analysis was carried out to study the efficiency of applying this concept and associated statistical data were obtained in a class of 40 students. A trend was observed regarding the class grade average throughout the entire semester. This trend is shown in Table 2 which expresses a general progress of students by looking at the class average. A similar trend was observed regarding the grades of individual students.

Table 2. Class average throughout the semester

\begin{tabular}{lc}
\hline Test or Quiz & Class Average \\
\hline First midterm & 70.48 \\
\hline Second midterm & 79.98 \\
\hline Third midterm & 83.22 \\
\hline Final & 84.15 \\
\hline
\end{tabular}


The benefits from implementing such a concept,

a- Isolate students' specific gaps in misunderstanding basic concepts.

b- Help them focus on remedy rather than shortcomings.

c- Individualize learning.

d- Help instructor tailor lectures to address specific gaps in understanding of concepts.

e- Ensure that learning is a two way process.

\section{V-Conclusions}

The current approach for teaching Aerospace Structures serves many purposes and has many advantages for students, academic institute, instructor, and TAs. It is presented in a generalized form hence it can be adapted to other Engineering courses. This generality comes from building its foundation onto two general concepts, Problem-Based Learning applied in the form of weekly problem sessions, Test Evaluation Concept and tailored work-plan. These concepts complement each other to form a learning environment where students focus on recognizing gaps in their understanding and working to remedy them. The main purpose of this approach is to make learning an attitude as well as an objective.

This approach prepares TAs to become well qualified instructors by allowing them the chance to be learners and teachers at the same time. The interaction occurring between them and the students together with getting the opportunity to contribute in the process of test evaluation exposes them to the broad concept of learning.

\section{VI- References}

1- Spense, L., "Maybe Teaching is a Bad Idea", Cutting ED, publication of James Madison University, Department of Integrated Science and Technology, issue- Winter 03/04. URL: www.isat.jmu.edu/cuttinged/ (Last accessed March 2006 )

2- Marshall, J., Marshall, J. "Innovative Teaching and Learning Strategies", American Society for Engineering Education Conference Proceedings. Nashville, TN, 2003.

3- Goankar, R., "Teaching Lessons from Engineering Feedback Model for New Educators", American Society for Engineering Education Conference Proceedings. Nashville, TN, 2003.

4- Evenson, D., Hmelo, Cindy (Editors) "Problem-Based Learning - a Research Perspective on Learning Interactions" Lawrence Erlbaum Associates, Inc., (2000)

5- Sharp, D., "Structural Loads Applied to Aircraft from Wing Pylons", Presented at Daniel Guggenheim School of Aerospace Engineering, Georgia Institute of Technology, Atlanta, GA, September 13, 2004. 